\title{
Dyadic Analysis of Partially Coherent Submillimeter-Wave Antenna Systems
}

\author{
Stafford Withington, Member, IEEE, Ghassan Yassin, Member, IEEE, and J. Anthony Murphy, Member, IEEE
}

\begin{abstract}
We describe a procedure for simulating the behavior of partially coherent submillimeter-wave antenna systems. The procedure is based on the principle that the second-order statistical properties of any partially coherent vector field can be decomposed into a sum of fully coherent, but completely uncorrelated, natural modes. Any of the standard electromagnetic analysis techniques-physical optics, geometrical theory of diffraction, etc.-can be used to propagate and scatter the modes individually, and the statistical properties of the total transformed field reconstructed at the output surface by means of superposition. In the case of modal optics-plane waves, Gaussian optics, waveguide mode matching, etc.- the properties of the field can be traced directly by means of scattering matrices. The overall procedure is of considerable value for calculating the behavior of astronomical instruments comprising planar and waveguide multimode bolometers, submillimeter-wave optical components, and large reflecting antennas.
\end{abstract}

Index Terms-Partial coherence, submillimeter-wave antennas.

\section{INTRODUCTION}

$\mathbf{T}$ HERE IS a considerable amount of interest in modeling the behavior of partially coherent submillimeter-wave antenna systems. Access to suitable techniques is particularly important in astronomy, where multimode bolometers are often placed in the focal planes of large reflecting antennas. Unfortunately, the techniques that are currently used for designing instruments of this kind are rather simplistic (ray tracing, etc.) and only provide a first-order description of behavior. This level of sophistication is not sufficient for the new high-performance ground-based and space-borne astronomical telescopes that are currently being planned. For example, there is an interest in using bolometers to determine the state of polarization of radiation from astronomical sources [1]. Most sources are polarized at an extremely low level, and great care has to be taken, at the design stage, to understand the intrinsic behavior of the telescope. The cross-polar scattering of antennas, and their associated mechanical structures, can only be modeled accurately by using full electromagnetic simulations.

The bolometers [2] used in astronomical instruments have a variety of forms. At one extreme, a heat-sensitive element is placed in a section of single-mode waveguide, which is coupled to the telescope by means of a waveguide horn [3]-[8]. At the other extreme, a planar bolometer is placed in the focal plane, and cold apertures are used to determine the throughput [9], [10]. Both of these arrangements are often packed into

Manuscript received March 27, 2000; revised January 16, 2001.

The authors are with the Cavendish Laboratory, Astrophysics Group, Cambridge CB3 OHE U.K. (e-mail: stafford@mrao.cam.ac.uk).

Publisher Item Identifier S 0018-926X(01)06372-4. imaging arrays. In the case of single-mode bolometers and extended sources, the throughput of the telescope is not being used efficiently, as there are many thermodynamic modes available for coupling power from the source to the detector. In the case of planar bolometers, the individual detectors are sensitive to radiation approaching from large angles, and stray light becomes a problem. A compromise is to place the detector in a few-moded waveguide and couple the detector to the telescope by means of an overmoded horn [11]-[13]. The short-wavelength version of this arrangement is the Winston cone [14], [15]. Whatever the physical realization, the design of a detector is always a compromise among angular resolution, sampling, throughput, and stray-light coupling.

To design systems, we would like to have an algorithm that can trace radiation from the source through the telescope onto the detector. At the same time, we would like to be able to assess the level of stray-light coupling. Ideally, we would like to carry out these calculations using the full range of classical electromagnetic techniques that are available for simulating the behavior of coherent fields. That is to say, in the case of overmoded waveguide bolometers, we would like to use mode-matching for the waveguide and horn [16], [17]; in the case of free-space diffraction, we would like to use plane waves, spherical harmonics, and Gaussian modes [18], [19]; and in the case of reflectors, we would like to use physical optics and the geometrical and physical theory of diffraction.

In this paper, we describe a procedure for modeling the behavior of partially coherent submillimeter-wave antenna systems. We explain how the statistical properties of any partially coherent vector field can be decomposed into a sum of natural modes. These modes are fully spatially coherent with respect to themselves but fully spatially incoherent with respect to each other. As a consequence, any of the standard electromagnetic analysis techniques can be used to propagate and scatter the modes individually, and then the statistical properties of the total transformed field reconstructed at the output surface by means of superposition. In the case of modal optics, the properties of the field can be traced directly by means of scattering matrices.

\section{Statistical Vector FieldS}

Before discussing submillimeter-wave systems, it is useful to formulate a general procedure for propagating and scattering the second-order statistical properties of partially coherent vector fields. Partially coherent scalar fields have been studied extensively in the optics literature [20], but partially coherent vector fields, where the state of coherence and polarization can vary over the region of interest, have received less attention. Often, 
when the vector properties of a field are included, it is assumed that degree of coherence and polarization do not vary as a function of position. A full description of partially coherent vector fields does exist, but this complex method, which defines electric, magnetic, and mixed coherence matrices [21], does not lend itself easily to the analysis of submillimeter-wave systems, nor does it provide much physical insight into the way long-wavelength optical systems behave. What is required is a formalism that is similar to the scalar methods of classical optics, in that the statistical properties of the field can vary as a function of position, but that does not introduce the full complexity of describing the $E$ and $H$ fields, and the correlations between them, separately. The scheme described achieves this balance.

To begin, we shall establish a formalism for describing the correlations between all three components of a vector electromagnetic field. We shall then assume that we are only interested in the properties of fields across simple surfaces: for example, we may wish to relate the focal-plane field of a large reflecting antenna to its spherical far-field antenna pattern. This assumption allows us to work in terms of two-dimensional tensors rather than three-dimensional tensors and simplifies the analysis considerably without compromising the utility of the technique.

To analyze the behavior of a field, we set up an ensemble of identical systems. In the spirit of analytic signal theory, we assume that the bandwidth of the measurement system is sufficiently narrow that the relative phases of field components at different points in any given member of the ensemble are well defined. The statistical properties enter through ensemble averages. In what follows, we do not refer to frequency explicitly.

The total electric field associated with any given member of the ensemble can be written

$$
\bar{E}(\bar{r})=E_{x}(\bar{r}) \hat{x}+E_{y}(\bar{r}) \hat{y}+E_{z}(\bar{r}) \hat{z}
$$

where $\bar{r}$ is a position vector and $\hat{x}, \hat{y}$, and $\hat{z}$ are orthogonal unit vectors that are defined throughout the volume of the field.

We can now define the cross-spectral dyadic as the dyadic product of the field at one point $\bar{r}$ and the complex conjugate of the field at a different point $\bar{r}^{\prime}$, averaged over the ensemble. The individual terms in the dyadic are the cross-spectral power densities of field components at two positions

$$
\overline{\bar{V}}\left(\bar{r}^{\prime} ; \bar{r}\right)=\left\langle\bar{E}(\bar{r}) \bar{E}^{*}\left(\bar{r}^{\prime}\right\rangle\right.
$$

which is analogous to the cross-spectral density of scalar fields. According to this scheme, the cross-correlations between field components are represented by an operator, $\overline{\bar{V}}\left(\bar{r}^{\prime} ; \bar{r}\right)$. This operator can be regarded as describing an intrinsic property of the field, and as such is independent of the particular basis set used.

We now choose a surface, which passes through the region of interest, that we wish to use as the source. On this surface, we establish an orthogonal triad of unit vectors, with two vectors tangential to the surface, $\hat{u}$ and $\hat{v}$, and one perpendicular, $\hat{w}$. At each point, this new system of vectors will in general be rotated with respect to the original system that was used to describe the cross-spectral dyadic $\overline{\bar{V}}\left(\bar{r}^{\prime} ; \bar{r}\right)$. Taking each member of the ensemble separately, we can project the field $\bar{E}(\bar{r})$ onto the vectors on the surface, giving $\bar{E}^{\prime}(\bar{r})$, where

$$
\bar{E}^{\prime}(\bar{r})=\overline{\bar{T}}(\bar{r}) \cdot \bar{E}(\bar{r})
$$

The nine components of the tensor $\overline{\bar{T}}(\bar{r})$ can be described in terms of the direction cosines relating the two basis sets. If we now define the cross-spectral dyadic over the surface, in the rotated coordinate system, by

$$
\overline{\bar{W}}\left(\bar{r}^{\prime} ; \bar{r}\right)=\left\langle\bar{E}^{\prime}(\bar{r}) \bar{E}^{\prime *}\left(\bar{r}^{\prime}\right)\right\rangle
$$

then after substituting (3), we find

$$
\overline{\bar{W}}\left(\bar{r}^{\prime} ; \bar{r}\right)=\overline{\bar{T}}(\bar{r}) \cdot \overline{\bar{V}}\left(\bar{r}^{\prime} ; \bar{r}\right) \cdot \overline{\bar{T}}^{* T}\left(\bar{r}^{\prime}\right)
$$

where $\overline{\bar{W}}\left(\bar{r}^{\prime} ; \bar{r}\right)$ is the cross-spectral dyadic in the new basis set. For reasons that will be described later, we will now ignore the component that is perpendicular to the surface and assume that $\overline{\bar{W}}\left(\bar{r}^{\prime} ; \bar{r}\right)$ is two-dimensional. In other words, we can always project the correlations between the vector components of a field onto a surface and extract those components that are tangential to the surface. This surface will be our source.

For convenience, the elements of the cross-spectral dyadic of the tangential field can be ordered into a matrix having components

$$
\begin{aligned}
& W_{u u}\left(\bar{r}^{\prime} ; \bar{r}\right)=\left\langle E_{u}^{\prime}(\bar{r}) E_{u}^{\prime *}\left(\bar{r}^{\prime}\right)\right\rangle \\
& W_{u v}\left(\bar{r}^{\prime} ; \bar{r}\right)=\left\langle E_{u}^{\prime}(\bar{r}) E_{v}^{\prime *}\left(\bar{r}^{\prime}\right)\right\rangle \\
& W_{v u}\left(\bar{r}^{\prime} ; \bar{r}\right)=\left\langle E_{v}^{\prime}(\bar{r}) E_{u}^{\prime *}\left(\bar{r}^{\prime}\right)\right\rangle \\
& W_{v v}\left(\bar{r}^{\prime} ; \bar{r}\right)=\left\langle E_{v}^{\prime}(\bar{r}) E_{v}^{\prime *}\left(\bar{r}^{\prime}\right)\right\rangle .
\end{aligned}
$$

According to the definition, we see that the cross-spectral dyadic is Hermitian: $\overline{\bar{W}}\left(\bar{r}^{\prime} ; \bar{r}\right)=\overline{\bar{W}}\left(\bar{r} ; \bar{r}^{\prime}\right)^{* T}$, where $* T$ denotes the conjugate transpose, a property that is of considerable importance.

We shall now make the approximation that for linear systems we can relate the field at a secondary surface $S_{2}$ to the field at a primary surface $S_{1}$, through an expression of the form

$$
\bar{E}_{2}\left(\bar{r}_{2}\right)=\int_{S_{1}} \overline{\bar{T}}\left(\bar{r}_{2} ; \bar{r}_{1}\right) \cdot \bar{E}_{1}\left(\bar{r}_{1}\right) d S_{1}
$$

where $\overline{\bar{T}}\left(\bar{r}_{2} ; \bar{r}_{1}\right)$ is a tensor propagator of some form. Also, $\bar{r}_{1} \in$ $S_{1}$ and $\overline{r_{2}} \in S_{2}$. This expression is completely general and can be used in cases where the propagator, sometimes called the point-spread function, but which now includes polarization, is a function of position.

Strictly speaking, it is not possible to propagate an electromagnetic field from one surface to another by using (7). The problem is that information is required about both the tangential electric and magnetic fields, or equivalently, the electric field and its normal derivative on the primary surface. In optical analysis, however, the propagator is often chosen to reduce the computational burden, and in many cases, the approximate solution based on the tangential electric field alone is almost indistinguishable from the rigorous solution based on a full analysis. 
In the case of bolometers and warm radiating surfaces, the situation is, in some ways, more favorable, because it is the surface current $\bar{J}_{1}\left(\bar{r}_{1}\right)$ that we wish to use as the source. In the case where there are no actual or equivalent magnetic surface currents on $S_{1}$, we have

$$
\bar{E}_{2}\left(\bar{r}_{2}\right)=\int_{S_{1}} \overline{\bar{G}}\left(\bar{r}_{2} ; \bar{r}_{1}\right) \cdot \bar{J}_{1}\left(\bar{r}_{1}\right) d S_{1}
$$

where $\overline{\bar{G}}\left(\bar{r}_{2} ; \bar{r}_{1}\right)$ is essentially the space-domain electric dyadic Green's function. Indeed, it seems that the dyadic formalism used so extensively in classical antenna and scattering problems [22] is closely related to the more general partially coherent representation described here. In practice, the difficulty associated with using (8) for a bolometer is that the Green's function must include the interface between the conducting surface and free space, and this generally means that impedance discontinuities are present. We shall return to this point later.

Regardless of the precise physical interpretation of the kernel-propagator or Green's function-we can substitute the expression for the field at the secondary surface [(7)] into the definition of the cross-spectral dyadic [(4)] to get an integral transform that relates the correlations

$$
\begin{aligned}
\overline{\bar{W}}_{2}\left(\bar{r}_{2}^{\prime} ; \bar{r}_{2}\right)= & \int_{S_{1}} \int_{S_{1}^{\prime}} \overline{\bar{T}}\left(\bar{r}_{2} ; \bar{r}_{1}\right) \cdot \overline{\bar{W}}_{1}\left(\bar{r}_{1}^{\prime} ; \bar{r}_{1}\right) \\
& \cdot \overline{\bar{T}}^{* T}\left(\bar{r}_{2}^{\prime} ; \bar{r}_{1}^{\prime}\right) d S_{1}^{\prime} d S_{1} .
\end{aligned}
$$

A similar expression could be derived using (8). For an aplanatic system, including polarization, we would have $\overline{\bar{T}}\left(\bar{r}_{2} ; \bar{r}_{1}\right) \equiv$ $\overline{\bar{T}}\left(\bar{r}_{2}-\bar{r}_{1}\right)$. The above expression describes the propagation of the statistical properties of a partially coherent vector field and is similar in form to its scalar analog.

In certain circumstances, there is a one-to-one mapping between the field on the primary surface and the field on the secondary surface-for example, when there is a free-space geometrical projection through an optical device such as a filter or polarizing grid. In this case, the transform becomes

$$
\bar{E}_{2}\left(\bar{r}_{2}\right)=\overline{\bar{T}}\left(\bar{r}_{2} ; \bar{r}_{1}\right) \cdot \bar{E}_{1}\left(\bar{r}_{1}\right)
$$

which, of course, is essentially the same as (3), but now the components of $\overline{\bar{T}}\left(\bar{r}_{2} ; \bar{r}_{1}\right)$ are determined by the physical properties of the device. We then have

$$
\overline{\bar{W}}_{2}\left(\bar{r}_{2}^{\prime} ; \bar{r}_{2}\right)=\overline{\bar{T}}\left(\bar{r}_{2} ; \bar{r}_{1}\right) \cdot \overline{\bar{W}}_{1}\left(\bar{r}_{1}^{\prime} ; \bar{r}_{1}\right) \cdot \overline{\bar{T}}^{* T}\left(\bar{r}_{2}^{\prime} ; \bar{r}_{1}^{\prime}\right) .
$$

To use (9) or (11), it is necessary to know the cross-spectral dyadic of the field over the primary surface. If there is some region in which it can be assumed that the source is fully incoherent, the three-dimensional cross-spectral dyadic takes the form

$$
\overline{\bar{V}}\left(\bar{r}^{\prime} ; \bar{r}\right)=\overline{\bar{I}} I(\bar{r}) \delta\left(\bar{r}-\bar{r}^{\prime}\right)
$$

where $I(\bar{r})$ is a measure of the strength of the field and $\overline{\bar{I}}$ is the idem factor.
If we now project this source field onto a surface that passes through the region occupied by the source, in the same way as described by (5), and make use of the identity

$$
\overline{\bar{T}}(\bar{r}) \cdot \overline{\bar{T}}^{* T}(\bar{r})=\overline{\bar{I}}
$$

we find that the tangential components of the field on the surface are uncorrelated

$$
\overline{\bar{W}}\left(\bar{r}^{\prime} ; \bar{r}\right)=\overline{\bar{I}} I(\bar{r}) \delta\left(\bar{r}-\bar{r}^{\prime}\right) .
$$

In other words, there are no correlations whatever the basis set.

By substituting (14) into the propagation integral (9), we can derive an expression for the field at the output of an optical system when the input is illuminated by an incoherent source, possibly of finite size. Furthermore, if we are only interested in the intensity and degree of polarization of the scattered field, it is sufficient to set $\bar{r}_{2}^{\prime}=\bar{r}_{2}$ to yield

$$
\overline{\bar{P}}_{2}\left(\bar{r}_{2}\right)=\int_{S_{1}} I_{1}\left(\bar{r}_{1}\right) \overline{\bar{K}}\left(\bar{r}_{2} ; \bar{r}_{1}\right) d S_{1}
$$

where $\overline{\bar{P}}(\bar{r})=\overline{\bar{W}}(\bar{r} ; \bar{r})$, and the kernel

$$
\overline{\bar{K}}\left(\bar{r}_{2} ; \bar{r}_{1}\right)=\left[\overline{\bar{T}}\left(\bar{r}_{2} ; \bar{r}_{1}\right) \cdot \overline{\bar{T}}^{* T}\left(\bar{r}_{2} ; \bar{r}_{1}\right)\right]
$$

is a dyadic that describes completely the propagation and scattering of incoherent sources. It is closely related to the kernel that is used to scatter fully coherent fields. Notice that the crossspectral dyadic at the output is Hermitian, $\bar{P}_{2}\left(\bar{r}_{2}\right)=\overline{\bar{P}}_{2}^{* T}\left(\bar{r}_{2}\right)$, as required.

The above expression confirms that if there is no cross-polar scattering

$$
\overline{\bar{T}}\left(\bar{r}_{2} ; \bar{r}_{1}\right)=\left[T_{u u}\left(\bar{r}_{2} ; \bar{r}_{1}\right) \hat{u} \hat{u}+T_{v v}\left(\bar{r}_{2} ; \bar{r}_{1}\right) \hat{v} \hat{v}\right]
$$

the orthogonal components of the field remain uncorrelated, and the statistical properties of each polarization can be treated separately

$$
\overline{\bar{K}}\left(\bar{r}_{2} ; \bar{r}_{1}\right)=T_{u u}^{2}\left(\bar{r}_{2} ; \bar{r}_{1}\right) \hat{u} \hat{u}+T_{v v}^{2}\left(\bar{r}_{2} ; \bar{r}_{1}\right) \hat{v} \hat{v} .
$$

In general, even free-space propagation leads to cross-polar coupling, the exception being paraxial optics, where the diffraction integral factorizes.

The eigenfunctions of transmission $\bar{\Psi}_{i}^{\alpha}(\bar{r})$ and the associated eigenvalues $\alpha_{i}$ are of particular importance. Considering (7), we look for solutions of the form

$$
\alpha_{i} \bar{\Psi}_{i}^{\alpha}\left(\bar{r}_{2}\right)=\int_{S_{1}} \overline{\bar{T}}\left(\bar{r}_{2} ; \bar{r}_{1}\right) \cdot \bar{\Psi}_{i}^{\alpha}\left(\bar{r}_{1}\right) d S_{1}
$$

where subscript $i$ is used to denote a particular eigenfunction and its eigenvalue.

In the case of scalar integral transforms, Mercer's theorem states that if the kernel of a homogeneous Fredholm equation of the second kind is Hermitian and nonnegative definite, then the eigenvalue spectrum is discrete and real and the eigenvectors form a complete orthonormal set in terms of which the kernel 
can be expanded. Hence it is reasonable to expect that the dyadic Green's function can be expanded as a series

$$
\overline{\bar{T}}\left(\bar{r}_{2} ; \bar{r}_{1}\right)=\sum_{i} \alpha_{i} \bar{\Psi}_{i}^{\alpha}\left(\bar{r}_{2}\right) \bar{\Psi}_{i}^{\alpha *}\left(\bar{r}_{1}\right)
$$

which can be seen to be plausible if we substitute (20) into the eigenfunction equation (19) and use the orthonormality condition

$$
\int_{S} \bar{\Psi}_{i}^{*}(\bar{r}) \cdot \bar{\Psi}_{j}(\bar{r}) d S=\delta_{i j}
$$

It can also be shown that for any set of vector fields to be complete, we require

$$
\sum_{i} \bar{\Psi}_{i}(\bar{r}) \bar{\Psi}_{i}^{*}\left(\bar{r}^{\prime}\right)=\overline{\bar{I}} \delta\left(\bar{r}-\bar{r}^{\prime}\right)
$$

an equality which will be used later.

The eigenfunctions introduced above are, of course, the natural modes of the optical system. That is to say, they are those vector fields that remain unchanged in functional form after having passed through the system. In reality, they may "look" very different depending on the coordinate systems used. This invariance does not mean that the fields pass through the system without being scattered; it simply means that they are scattered in such a way that, at the secondary surface, they have returned to their original forms.

Given that the propagator $\overline{\bar{T}}\left(\bar{r}_{2} ; \bar{r}_{1}\right)$ can be described in terms of its eigenfunctions [(20)], it is tempting to expand the crossspectral dyadic in terms of a set of orthogonal vector functions. Indeed, we might expect the cross-spectral dyadic to have an expansion of the form

$$
\overline{\bar{W}}\left(\bar{r}^{\prime} ; \bar{r}\right)=\sum_{i} \beta_{i} \bar{\Psi}_{i}^{\beta}(\bar{r}) \bar{\Psi}_{i}^{\beta *}\left(\bar{r}^{\prime}\right)
$$

where

$$
\beta_{i} \bar{\Psi}_{i}^{\beta}(\bar{r})=\int_{S^{\prime}} \overline{\bar{W}}\left(\bar{r}^{\prime} ; \bar{r}\right) \cdot \bar{\Psi}_{i}^{\beta}\left(\bar{r}^{\prime}\right) d S^{\prime} .
$$

It is clear from the definition of the cross-spectral dyadic [(4)] that if a partially coherent field is formed from a superposition of fields that are fully incoherent with respect to each other, whatever the states of coherence of the individual fields, the composite cross-spectral dyadic is given by the sum

$$
\overline{\bar{W}}\left(\bar{r}^{\prime} ; \bar{r}\right)=\sum_{i} \overline{\bar{W}}_{i}\left(\bar{r}^{\prime} ; \bar{r}\right) .
$$

The expansion of the cross-spectral dyadic into its set of eigenfunctions therefore describes the process of decomposing a partially coherent vector field into a sum of fields that are fully incoherent with respect to each other. The set of vector fields $\bar{\Psi}_{i}^{\beta}(\bar{r})$ are the natural modes of the field, as distinct from the natural modes of the optical system $\bar{\Psi}_{i}^{\alpha}(\bar{r})$.

Generally, of course, the natural modes of the field are not the same as those of the optical system; there is, however, one exception. If we assume that the primary field is fully incoherent and effectively infinite in size, and use the completeness relation (22) over the set of system eigenfunctions, we can write

$$
\overline{\bar{W}}\left(\bar{r}^{\prime} ; \bar{r}\right)=\overline{\bar{I}} \delta\left(\bar{r}-\bar{r}^{\prime}\right)=\sum_{i} \bar{\Psi}_{i}^{\alpha}(\bar{r}) \bar{\Psi}_{i}^{\alpha *}\left(\bar{r}^{\prime}\right) .
$$

We can then substitute this expression into the propagation integral $[(9)]$ to show that for any system, the natural modes of the field at the secondary surface are the eigenfunctions of the system. In other words, all aspects of the fields coherence are determined by the system. This behavior is to be expected simply from the point of view of mode filtering. It shows, for example, that in waveguide problems, all modes are excited equally and incoherently when a blackbody source fills the waveguide.

\section{MATRIX REPRESENTATION OF PARTIALLy COHERENT SYSTEMS}

To give the scheme practical value, we need to express the above forms in terms of some particular representation. Notice that we have already assumed that the eigenvalue spectrum is discrete. In other words, the field at the primary surface is of limited extent and its spatial spectrum is limited also. This situation will prevail if the primary surface has been illuminated by an optical system having finite throughput. It will also be the case if the angular range over which we wish to determine the properties of the radiated field is restricted. A description of optical-field sampling is provided by many texts [23], [24].

We can expand the field associated with each member of the ensemble in terms of a set of vector fields that are appropriate to the region. These are not necessarily the natural modes of the field but can be any complete orthonormal set. For example, they may be the TE and TM modes of metallic waveguide, a set of polarized plane waves, or a set of polarized spherical waves. Whatever the choice, we can write

$$
\bar{E}(\bar{r})=\sum_{n} A_{n} \bar{\Psi}_{n}(\bar{r})
$$

where the modes are described by

$$
\bar{\Psi}_{n}(\bar{r})=\psi_{n}(\bar{r}) \hat{u}+\phi_{n}(\bar{r}) \hat{v}
$$

and $\psi_{n}(\bar{r})$ and $\phi_{n}(\bar{r})$ are the individual field components. Here, we use a single index to represent what is usually a double index. In other words, we label every two-dimensional mode with a single unique identifier.

Substituting the field expansion (27) into the definition of the cross-spectral dyadic [(4)], we find that the statistical properties of the field are represented by a set of coefficients $C_{n m}$, where

$$
\overline{\bar{W}}\left(\bar{r}^{\prime} ; \bar{r}\right)=\sum_{n m} C_{n m} \bar{\Psi}_{n}(\bar{r}) \bar{\Psi}_{m}^{*}\left(\bar{r}^{\prime}\right)
$$

and

$$
C_{n m}=\left\langle A_{n} A_{m}^{*}\right\rangle .
$$

For convenience, we can order the coefficients of this bimodel expansion into a matrix, which we shall call the coherence matrix $\mathbf{C}$. 
Once the coherence matrix is known, the elements of the cross-spectral dyadic are given by

$$
\begin{aligned}
& W_{u u}\left(\bar{r}^{\prime} ; \bar{r}\right)=\sum_{n m} C_{n m} \psi_{n}(\bar{r}) \psi_{m}^{*}\left(\bar{r}^{\prime}\right) \\
& W_{u v}\left(\bar{r}^{\prime} ; \bar{r}\right)=\sum_{n m} C_{n m} \psi_{n}(\bar{r}) \phi_{m}^{*}\left(\bar{r}^{\prime}\right) \\
& W_{v u}\left(\bar{r}^{\prime} ; \bar{r}\right)=\sum_{n m} C_{n m} \phi_{n}(\bar{r}) \psi_{m}^{*}\left(\bar{r}^{\prime}\right) \\
& W_{v v}\left(\bar{r}^{\prime} ; \bar{r}\right)=\sum_{n m} C_{n m} \phi_{n}(\bar{r}) \phi_{m}^{*}\left(\bar{r}^{\prime}\right) .
\end{aligned}
$$

where the summations extend over all modes.

At the output surface, we are usually only interested in correlations between field components at a point, in which case $\bar{r}^{\prime}=\bar{r}$, and the cross-spectral dyadic reduces to a simple tensor field, which is essentially the classical polarization matrix

$$
\overline{\bar{P}}(\bar{r})=\overline{\bar{W}}(\bar{r} ; \bar{r}) .
$$

Hence, we can find Stokes parameters at any point [25]

$$
\begin{aligned}
& S_{0}(\bar{r})=\left[P_{u u}(\bar{r})+P_{v v}(\bar{r})\right] \\
& S_{1}(\bar{r})=\left[P_{u u}(\bar{r})-P_{v v}(\bar{r})\right] \\
& S_{2}(\bar{r})=\left[P_{u v}(\bar{r})+P_{v u}(\bar{r})\right] \\
& S_{3}(\bar{r})=j\left[P_{u v}(\bar{r})-P_{v u}(\bar{r})\right] .
\end{aligned}
$$

In summary, once the coherence matrix is known for the surface of interest, we can derive all the commonly used measures of behavior, including the degree of polarization, the nature of the polarization, and the average power flow.

Of particular interest is the power in the beam, which must be one of the invariants of lossless propagation. The power density is simply $I(\bar{r})=S_{0}(\bar{r})=\left[P_{u u}(\bar{r})+P_{v v}(\bar{r})\right]$, but we also have

$$
\begin{aligned}
& P_{u u}(\bar{r})=\sum_{n m} C_{n m} \psi_{n}(\bar{r}) \psi_{m}^{*}(\bar{r}) \\
& P_{v v}(\bar{r})=\sum_{n m} C_{n m} \phi_{n}(\bar{r}) \phi_{m}^{*}(\bar{r}) .
\end{aligned}
$$

We can integrate over the surface to get the total power flow $P_{t}$, which gives

$$
P_{t}=\int_{S} I(\bar{r}) d S=\sum_{n m} C_{n m} \delta_{n m}=\operatorname{Tr} \mathbf{C} .
$$

Hence the total power is simply the trace of the coherence matrix; it is also the trace of the polarization matrix.

We need a procedure for finding the elements of the coherence matrix when the elements of the cross-spectral dyadic are known. It can be shown that

$$
C_{n m}=\int_{S} \int_{S^{\prime}} \bar{\Psi}_{n}^{*}(\bar{r}) \cdot \overline{\bar{W}}\left(\bar{r}^{\prime} ; \bar{r}\right) \cdot \bar{\Psi}_{m}\left(\bar{r}^{\prime}\right) d S^{\prime} d S
$$

which can be appreciated by substituting the bimodal expansion (29) into (36) and using the orthonormality condition. Hence, we can find the coherence matrix for any vector basis set once the cross-spectral dyadic is known.
Usually the field over the source is either fully coherent or fully incoherent, and then the above equation takes on two special forms. When the field is fully incoherent, we have

$$
C_{n m}=\int_{S} I(\bar{r})\left[\psi_{n}^{*}(\bar{r}) \psi_{m}(\bar{r})+\phi_{n}^{*}(\bar{r}) \phi_{m}(\bar{r})\right] d S .
$$

When the field is fully coherent, we can find the set of mode coefficients through

$$
A_{n}=\int_{S} \bar{E}(\bar{r}) \cdot \bar{\Psi}_{n}^{*}(\bar{r}) d S
$$

and then form the coherence matrix by

$$
\mathbf{C}=\mathbf{A A}^{* T}
$$

where $\mathbf{A}$ is the column vector of mode coefficients.

It is always possible to decompose a field into fully coherent and fully incoherent parts, and often the physical significance of the decomposition is clear. When the total field is constructed from a number of fields that are fully incoherent with respect to each other, regardless of the state of coherence of each contribution, the overall coherence matrix is given by

$$
\mathbf{C}=\sum_{i} \mathbf{C}_{i}
$$

where $\mathbf{C}_{i}$ are the individual coherence matrices. When simulating the behavior of optical systems, it is convenient to be able to assemble composite coherence matrices in this way.

Finally, it is important to be able to propagate and scatter coherence matrices. There are various ways in which this can be achieved depending on the nature of the problem. If we know the scattering matrix associated with the basis set, the solution is straightforward; for we have $\mathbf{B}=\mathbf{S A}$, where $\mathbf{A}$ is the vector of mode coefficients on the primary surface, $\mathbf{B}$ is the vector of mode coefficients on the secondary surface, and $\mathbf{S}$ is the scattering matrix that relates them. We can then write

$$
\mathbf{D}=\left\langle\mathbf{B B}^{* T}\right\rangle=\mathbf{S C S}^{* T} .
$$

This approach is useful, for example, when modes are scattered in metallic waveguide, or when Gaussian-Hermite [19] or spherical modes are used for free-space propagation. In many cases, we need a more general method for scattering and propagating a partially coherent field; for example, when tracing a field through a reflector. There is an elegant way in which the well-known electromagnetic analysis techniques can be used for this purpose.

It can be seen that the coherence matrix is Hermitian. It is also straightforward to show that the coherence matrix is nonnegative: $\mathbf{Z}^{* T} \mathbf{C Z} \geq 0$ for all $\mathbf{Z}$, where $\mathbf{Z}$ is any complex column vector. Hence there is some unitary transformation $\mathbf{U}$ for which $\mathbf{U}^{* T} \mathbf{C U}=\boldsymbol{\Lambda}$, where the individual columns of $\mathbf{U}$ are the eigenvectors of the coherence matrix and $\boldsymbol{\Lambda}$ is a diagonal matrix of eigenvalues. Hence we can diagonalize the coherence matrix and then assemble the eigenfunctions through

$$
\bar{\Psi}_{i}^{\beta}\left(\bar{r}_{1}\right)=\sum_{j} U_{j i}\left[\psi_{j}\left(\bar{r}_{1}\right) \hat{u}+\phi_{j}\left(\bar{r}_{1}\right) \hat{v}\right] .
$$


These are the natural modes of the field discussed earlier. This decomposition represents the vector form of a scalar version, which has been investigated extensively by Wolf [26], [27].

We can decompose any partially coherent electromagnetic field into a sum of fully coherent, completely uncorrelated, natural modes. We can then scatter each of these elemental fields using the electromagnetic technique that is most suitable for the problem being considered. Let us say that after scattering, each elemental field maps onto some new distribution $\bar{\Psi}_{i}^{\beta}\left(\bar{r}_{1}\right) \rightarrow$ $\bar{\Psi}_{i}^{\gamma}\left(\bar{r}_{2}\right)$, where the coordinate system and basis vectors used for the primary and secondary surfaces may be different. Because the scattered fields are fully coherent but completely uncorrelated, it follows that

$$
\overline{\bar{W}}_{2}\left(\bar{r}_{2}^{\prime} ; \bar{r}_{2}\right)=\sum_{i} \beta_{i} \bar{\Psi}_{i}^{\gamma}\left(\bar{r}_{2}\right) \bar{\Psi}_{i}^{\gamma *}\left(\bar{r}_{2}^{\prime}\right)
$$

Or, if we are only interested in the correlations between field components at a point

$$
\overline{\bar{P}}\left(\bar{r}_{2}\right)=\sum_{i} \beta_{i} \bar{\Psi}_{i}^{\gamma}\left(\bar{r}_{2}\right) \bar{\Psi}_{i}^{\gamma *}\left(\bar{r}_{2}\right)
$$

The decomposition of a field into its natural modes will allow techniques such as physical optics and the geometrical theory of diffraction to be used for simulating the behavior of complex partially coherent long-wavelength optical systems. Also, as is shown in the Appendix, the entropy of a vector field can be determined easily once the coherence matrix is known. The possibility of using phase-retrieval maximum-entropy techniques to recover the coherence matrices of submillimeter-wave sources from intensity measurements alone is of particular interest [28].

\section{Analyzing a Circular-Waveguide Bolometer}

To illustrate the above procedure, we will consider a bolometer comprising a perfectly absorbing disc in a circular waveguide. At one end, the waveguide is terminated by a cold absorbing backshort, while at the other end, it tapers into a conical horn. By using a disc that does not completely fill the waveguide, we have made the bolometer difficult to analyze. If the disc filled the waveguide, all of the waveguide modes would be excited equally, and the solution would be straightforward to find. The bolometer described here is rather ideal, but it is possible by combining our formalism with traditional mode-matching methods to analyze much more complicated arrangements: overmoded corrugated horns, integrating cavities, cavities with supporting coaxial structures, Winston cones, etc.

To begin, we must find the elements of the coherence matrix represented in terms of a complete set of waveguide modes. If we take the $z$-axis to be the axis of the waveguide, of radius $a$, then the $z$-component of the $E$-field of the $\mathrm{TM}_{n l}$ mode, or $E$ mode, is given by

$$
E_{z}^{E}(r, \phi) \propto J_{n}\left(k_{c} r\right) e^{j n \phi}, \quad r \leq a
$$

where $k_{c}=p_{n l} / a$ is the cutoff wavenumber of the $n l$ th mode and $J_{n}\left(p_{n l}\right)=0$. Also, the $z$-component of the $H$-field of the $\mathrm{TE}_{n l}$ mode, or $H$ mode, is given by

$$
H_{z}^{H}(r, \phi) \propto J_{n}\left(k_{c}^{\prime} r\right) e^{j n \phi}, \quad r \leq a
$$

where $k_{c}^{\prime}=p_{n l}^{\prime} / a$ is the cutoff wavenumber of the $n l$ th mode and $J_{n}^{\prime}\left(p_{n l}^{\prime}\right)=0$. According to this scheme, the azimuthal index takes on values $-\infty<n<\infty$.

If we use a set of Cartesian vectors for the polarization, and following the notation introduced previously, we can write

$$
\begin{aligned}
\psi_{n l}^{H}(\bar{r}) & =N_{n l}\left[J_{n+1}\left(k_{c}^{\prime} r\right) e^{j \phi}+J_{n-1}\left(k_{c}^{\prime} r\right) e^{-j \phi}\right] e^{j n \phi} \\
\phi_{n l}^{H}(\bar{r}) & =N_{n l}\left[-J_{n+1}\left(k_{c}^{\prime} r\right) e^{j \phi}+J_{n-1}\left(k_{c}^{\prime} r\right) e^{-j \phi}\right] j e^{j n \phi} \\
\psi_{n l}^{E}(\bar{r}) & =M_{n l}\left[-J_{n+1}\left(k_{c} r\right) e^{j \phi}+J_{n-1}\left(k_{c} r\right) e^{-j \phi}\right] e^{j n \phi} \\
\phi_{n l}^{E}(\bar{r}) & =M_{n l}\left[J_{n+1}\left(k_{c} r\right) e^{j \phi}+J_{n-1}\left(k_{c} r\right) e^{-j \phi}\right] j e^{j n \phi}
\end{aligned}
$$

where the normalization constants are given by

$$
M_{n l}=\left[J_{n+1}\left(p_{n l}\right)\right]^{-1}
$$

for the $E$ modes and

$$
N_{n l}=\left[\left(1-\left(n / p_{n l}^{\prime}\right)^{2}\right)^{1 / 2} J_{n}\left(p_{n l}^{\prime}\right)\right]^{-1}
$$

for the $H$ modes. These modes form a complete set of functions in terms of which the field at any plane can be described.

Now suppose that we have some spatially incoherent source of radius $b$ in the waveguide. We can use (37) to calculate the elements of the coherence matrix at the source plane. In this case, the coherence matrix is a block matrix, the submatrices of which give the correlations between the different types of modes. Using (47), we find

$$
\begin{aligned}
C_{n l, m s}^{H H}= & N_{n l} N_{n s} \int_{0}^{b / a}\left[J_{n+1}\left(p_{n l}^{\prime} u\right) J_{n+1}\left(p_{n s}^{\prime} u\right)\right. \\
& \left.+J_{n-1}\left(p_{n l}^{\prime} u\right) J_{n-1}\left(p_{n s}^{\prime} u\right)\right] u d u \delta_{m, n} \\
C_{n l, m s}^{E E}= & M_{n l} M_{n s} \int_{0}^{b / a}\left[J_{n+1}\left(p_{n l} u\right) J_{n+1}\left(p_{n s} u\right)\right. \\
& \left.+J_{n-1}\left(p_{n l} u\right) J_{n-1}\left(p_{n s} u\right)\right] u d u \delta_{m, n} \\
C_{n l, m s}^{H E}= & N_{n l} M_{n s} \int_{0}^{b / a}\left[-J_{n+1}\left(p_{n l}^{\prime} u\right) J_{n+1}\left(p_{n s} u\right)\right. \\
& \left.+J_{n-1}\left(p_{n l}^{\prime} u\right) J_{n-1}\left(p_{n s} u\right)\right] u d u \delta_{m, n} \\
C_{n l, m s}^{E H}= & M_{n l} N_{n s} \int_{0}^{b / a}\left[-J_{n+1}\left(p_{n l} u\right) J_{n+1}\left(p_{n s}^{\prime} u\right)\right. \\
& \left.+J_{n-1}\left(p_{n l} u\right) J_{n-1}\left(p_{n s}^{\prime} u\right)\right] u d u \delta_{m, n} .
\end{aligned}
$$

It should be appreciated that an infinite number of modes is required to make the mode set complete. Only on propagation are modes filtered, and this filtering leads to spatial correlations and some degree of polarization. Even when the absorber completely fills the waveguide, the propagating field and the beams patterns are polarized. When the absorber has a finite size, correlations are induced between waveguide modes. Because the modes slip in phase with respect to each other, the form of the field changes on propagation. The beam pattern depends on the 
total length of the waveguide and horn. This behavior of course has nothing to do with standing waves, which is another issue.

Equation (50) is, strictly speaking, only an approximation. The problem is that in the case of lossy conductors, it is the surface current rather than the outwardly propagating surface field that is known. The outwardly propagating surface field depends on the surface impedance of the conductor and the impedance of the medium in which it is contained. When a bolometer is contained in a few-moded waveguide, those modes close to cutoff will have impedances significantly different from the $377 \Omega$ of those modes that are well above cutoff. Hence differential coupling will occur and "mode filtering" will take place. For multimoded waveguides, those modes well above cutoff will contain the majority of the power, and the effect of poor coupling into those modes close to cutoff will go unnoticed-although strictly speaking, the radiated field will lose some of its high spatial-frequency content. Multimoded systems, or free-space systems, will not suffer from this effect. It is interesting to note, however, that even when an integrating cavity is placed at the back of a waveguide, differential coupling will be present, and again some high spatial frequencies will be lost. In general terms, it is possible to include differential coupling in the formalism, but here, for brevity, we shall ignore this effect.

According to (41), we could propagate the coherence matrix by using the scattering matrix of the waveguide and horn. The scattering matrix could be calculated by means of the classical mode matching technique, but here, for simplicity, we shall assume that there is no intermodal scattering, which is a good approximation for small flare angles. The scattering matrix then becomes

$$
S_{n l, n^{\prime} l^{\prime}}=e^{-j \phi_{n l}} \delta_{n n^{\prime}} \delta_{l l^{\prime}}
$$

where

$$
\phi_{n l}=k \int_{z 1}^{z 2}\left[1-\left(\frac{\lambda}{\lambda c_{n l}(z)}\right)^{2}\right]^{1 / 2} d z
$$

$\lambda c_{n l}=2 \pi a(z) / p_{n l}$ for the $E$ modes, and $\lambda c_{n l}=2 \pi a(z) / p_{n l}^{\prime}$ for the $H$ modes. The scattering matrix is diagonal, and

$$
S_{n l, n^{\prime} l^{\prime}}=0
$$

for modes below cutoff, $\lambda>\lambda c_{n l} . k$ is the free-space propagation constant.

At the aperture of the horn, there are several options depending precisely on what we want to do. We may simply want to look at the aperture field, in which case we can reconstruct the cross-spectral dyadic through

$$
\begin{aligned}
\overline{\bar{W}}\left(\bar{r}^{\prime} ; \bar{r}\right)= & \sum_{n l, m s} C_{n l, m s}^{H H} \bar{\Psi}_{n l}^{H}(\bar{r}) \bar{\Psi}_{m s}^{H *}\left(\bar{r}^{\prime}\right) \\
& +\sum_{n l, m s} C_{n l, m s}^{E H} \bar{\Psi}_{n l}^{E}(\bar{r}) \bar{\Psi}_{m s}^{H *}\left(\bar{r}^{\prime}\right) \\
& +\sum_{n l, m s} C_{n l, m s}^{H E} \bar{\Psi}_{n l}^{H}(\bar{r}) \bar{\Psi}_{m s}^{E *}\left(\bar{r}^{\prime}\right) \\
& +\sum_{n l, m s} C_{n l, m s}^{E E} \bar{\Psi}_{n l}^{E}(\bar{r}) \bar{\Psi}_{m s}^{E *}\left(\bar{r}^{\prime}\right)
\end{aligned}
$$

where $\mathbf{C}$ is the coherence matrix after propagation and the functional forms of the modal components are given by (47). In this reconstruction, we have again taken into account the fact that the coherence matrix is a block matrix.

If we are interested in calculating the far-field radiation pattern of the horn, we could scatter the waveguide modes into Gaussian-Hermite modes, plane waves, or spherical harmonics. It is not sufficient, in the case of a bolometer that does not fill the waveguide, to simply radiate the individual waveguide modes and add them incoherently. Alternately, if we are illuminating a reflector, we may wish to use a physical optics algorithm of some kind. In this case, we diagonalize the coherence matrix at the aperture of the horn and reconstruct the eigenmodes, which are represented in terms of the set of waveguide modes. According to (42), if $U_{j i}$ is the $j$ th coefficient of the $i$ th eigenmode of $\mathbf{C}$, we have

$$
\bar{\Psi}_{i}^{\beta}(\bar{r})=\left[\sum_{j} U_{j i}^{E} \bar{\Psi}_{j}^{E}(\bar{r})+\sum_{j} U_{j i}^{H} \bar{\Psi}_{j}^{H}(\bar{r})\right] e^{-\left(k r^{2} / 2 L\right)}
$$

where we have reverted to using a single index to represent a two-dimensional mode, and again we have emphasized the block nature of the coherence matrix. We have also included a phase factor to account for the phase curvature of the field at the aperture: $r$ represents the radial position in the aperture and $L$ is the slant length. Each of these modes can now be propagated into the far field independently and the total field reconstructed on the output surface.

To calculate the far-field behavior of a free-standing horn, we can take the Fourier transform of each of the natural modes. If the $i$ th eigenmode has field components $\psi_{i}^{\beta}(\bar{r})$ and $\phi_{i}^{\beta}(\bar{r})$, or equivalently

$$
\bar{\Psi}_{i}^{\beta}(\bar{r})=\psi_{i}^{\beta}(\bar{r}) \hat{x}+\phi_{i}^{\beta}(\bar{r}) \hat{y}
$$

where now $\bar{r}$ corresponds to a point in the aperture, we can define the plane-wave spectra [30] according to

$$
\begin{aligned}
& F x_{i}(\bar{k})=\frac{1}{\lambda^{2}} \int_{S} \psi_{i}^{\beta}(\bar{r}) e^{-\left(k r^{2} / 2 L\right)} e^{j \bar{k} \cdot \bar{r}} d S \\
& F y_{i}(\bar{k})=\frac{1}{\lambda^{2}} \int_{S} \phi_{i}^{\beta}(\bar{r}) e^{-\left(k r^{2} / 2 L\right)} e^{j \bar{k} \cdot \bar{r}} d S .
\end{aligned}
$$

In these expressions, we have again included a phase factor to represent the phase curvature of the field at the aperture of the horn. Remember that we can describe the wavevector $\bar{k}$ in terms of the direction cosines $\alpha$ and $\beta: \bar{k}=k \alpha \hat{i}+k \beta \hat{j}$.

Each natural mode, as represented by its plane-wave spectrum, maps onto some far-field field distribution $\bar{\Psi}_{i}^{\beta}(\bar{r}) \rightarrow \bar{\Psi}_{i}^{\gamma}(\rho, \theta, \phi)$ where the field is now described in terms of spherical coordinates. This mapping is given by

$$
\bar{\Psi}_{i}^{\gamma}(\rho, \theta, \phi)=j 2 \pi \frac{e^{-j k \rho}}{k \rho}\left[E_{\theta i}(\theta, \phi) \hat{\theta}+E_{\phi i}(\theta, \phi) \hat{\phi}\right]
$$

where

$$
\begin{aligned}
E_{\theta i}(\theta, \phi)= & {\left[F x_{i}(\alpha, \beta) \cos \phi+F y_{i}(\alpha, \beta) \sin \phi\right] } \\
E_{\phi i}(\theta, \phi)=[ & -F x_{i}(\alpha, \beta) \cos \theta \sin \phi \\
& \left.\quad+F y_{i}(\alpha, \beta) \cos \theta \cos \phi\right]
\end{aligned}
$$

with $\alpha=\sin \theta \cos \phi$ and $\beta=\sin \theta \sin \phi$. 
As described by (43), the polarization matrix can then be reassembled through

$$
\overline{\bar{W}}\left(\rho, \theta, \phi ; \rho^{\prime}, \theta^{\prime}, \phi^{\prime}\right)=\sum_{i} \beta_{i} \bar{\Psi}_{i}^{\gamma}(\rho, \theta, \phi) \bar{\Psi}_{i}^{\gamma *}\left(\rho^{\prime}, \theta^{\prime}, \phi^{\prime}\right)
$$

but because we are only interested in correlations over a fixed sphere, $\rho=\rho^{\prime}$, we have

$$
\begin{aligned}
& \overline{\bar{W}}_{\theta \theta}\left(\theta, \phi ; \theta^{\prime}, \phi^{\prime}\right) \propto \sum_{i} \beta_{i} E_{\theta i}(\theta, \phi) E_{\theta i}^{*}\left(\theta^{\prime}, \phi^{\prime}\right) \\
& \overline{\bar{W}}_{\theta \phi}\left(\theta, \phi ; \theta^{\prime}, \phi^{\prime}\right) \propto \sum_{i} \beta_{i} E_{\theta i}(\theta, \phi) E_{\phi i}^{*}\left(\theta^{\prime}, \phi^{\prime}\right) \\
& \overline{\bar{W}}_{\phi \theta}\left(\theta, \phi ; \theta^{\prime}, \phi^{\prime}\right) \propto \sum_{i} \beta_{i} E_{\phi i}(\theta, \phi) E_{\theta i}^{*}\left(\theta^{\prime}, \phi^{\prime}\right) \\
& \overline{\bar{W}}_{\phi \phi}\left(\theta, \phi ; \theta^{\prime}, \phi^{\prime}\right) \propto \sum_{i} \beta_{i} E_{\phi i}(\theta, \phi) E_{\phi i}^{*}\left(\theta^{\prime}, \phi^{\prime}\right) .
\end{aligned}
$$

From these, we can find Stokes parameters over a spherical farfield surface.

\section{CONCLUSION}

We have described a procedure for simulating the behavior of partially coherent submillimeter-wave antenna systems. The technique is based on the principle that any partially coherent vector field can be decomposed into a sum of fully spatially coherent, but completely uncorrelated, natural modes. Standard electromagnetic analysis techniques can be used to propagate and scatter the modes individually, and then the statistical properties of the total transformed field can be reconstructed at the output surface by means of superposition.

The procedure has been illustrated by considering a waveguide bolometer. The problem was made intentionally awkward by using an absorbing disc that did not completely fill the waveguide. If the disc had filled the waveguide, all of the waveguide modes would have been excited incoherently and the problem would have been straightforward to solve.

The proposed scheme has many applications. In particular, we are interested in analyzing the behavior of planar and multimode-waveguide bolometers. We are also interested in using the technique to propagate surface roughness effects through sequences of terahertz reflectors, and to determine, through maximum entropy techniques, the state of partially coherent fields from intensity measurements alone.

\section{APPENDIX}

Here we show that the entropy of a vector field can be determined easily once the coherence matrix is known. The diagonalized coherence matrix corresponds to a superposition of natural modes, and each eigenvalue gives the power in each mode. The probability $p_{i}$ that a photon occupies the $i$ th eigenmode is given by

$$
p_{i}=\frac{\lambda_{i}}{\sum_{j} \lambda_{j}}
$$

and the entropy becomes

$$
Q=-\sum_{i} p_{i} \ln \left(p_{i}\right)
$$

Hence, by diagonalizing the coherence matrix, we can determine the entropy of the field [29]. In many cases, however, it is inconvenient and time-consuming to diagonalize large coherence matrices, and a more direct way of finding the entropy is required.

To this end, we remember that a function $\Phi(\mathbf{C})$ of a matrix $\mathbf{C}$ is defined in terms of its power series

$$
\Phi(\mathbf{C})=\sum_{i} \kappa_{i} \mathbf{C}^{i}
$$

where $\mathbf{C}^{0}=\mathbf{I}$. This series converges if the eigenvalues of $\mathbf{C}$ satisfy $\left|\lambda_{i}\right|<R$, where $R$ is the radius of convergence of the scalar form of the expansion. If we normalize the coherence matrix to its trace, $\mathbf{C}^{\prime}=\mathbf{C} / \operatorname{Tr} \mathbf{C}$, then all of the eigenvalues are less than unity and the power series of $\ln \left(\mathbf{C}^{\prime}\right)$ converges. We then consider

$$
-\left[\mathbf{C}^{\prime} \ln \left(\mathbf{C}^{\prime}\right)\right]=\mathbf{C}^{\prime} \sum_{r=1}^{r=\infty} \frac{1}{r}\left(\mathbf{I}-\mathbf{C}^{\prime}\right)^{r} .
$$

Now the normalized coherence matrix can be expressed as a sum of natural modes $\mathbf{C}_{i}^{\prime}$

$$
\mathbf{C}^{\prime}=\sum_{i=1}^{N} \lambda_{i} \mathbf{C}_{i}^{\prime}
$$

Also, by noting that

$$
\mathbf{C}_{i}^{\prime} \mathbf{C}_{j}^{\prime}=\mathbf{C}_{i}^{\prime} \delta_{i j}
$$

we find, after some manipulation, that

$$
Q=\operatorname{Tr}\left[\mathbf{C}^{\prime} \sum_{r=1}^{r=\infty} \frac{1}{r}\left(\mathbf{I}-\mathbf{C}^{\prime}\right)^{r}\right] .
$$

The entropy $Q$ is therefore found through simple matrix multiplications, and the first few terms of this expansion provide a convenient measure of the degree of disorder of a vector electromagnetic field.

\section{REFERENCES}

[1] R. H. Hildebrand et al., "Hertz, an imaging polarimeter," in Proc. SPIE Advanced Technology MMW, Radio, and THz Telescopes, vol. 3357, Kona, HI, Mar. 1998, pp. 289-296.

[2] P. L. Richards, "Bolometers for infrared and millimeter waves," J. Appl. Phys., vol. 76, no. 1, pp. 1-24, July 1994.

[3] W. K. Gear and C. R. Cunningham, "SCUBA: A camera for the James Clerk Maxwell telescope," in Conf. Multi-Feed Systems for Radio Telescopes, vol. 75, D. T. Emerson and J. M. Payne, Eds, May 1994, pp. 215-221.

[4] W. S. Holland et al., "SCUBA: A common-user submillimeter camera operating on the James Clerk Maxwell Telescope," Monthly Notices Roy. Astron. Soc.y, vol. 303, no. 4, pp. 659-672, 1999.

[5] J. Glenn et al., "Bolocam: A millimeter-wave bolometer camera," in Proc. SPIE Advanced Technology MMW, Radio, and THz Telescopes, vol. 3357, Kona, HI, Mar. 1998, pp. 326-334.

[6] E. Kreysa et al., "Bolometer array development at the Max-Planck Institut fur Radioastronomie," in Proc. SPIE Advanced Technology MMW, Radio, and THz Telescopes, vol. 3357, Kona, HI, Mar. 1998, pp. 319-325.

[7] E. Kreysa et al., "Bolometer array development at the Max-Planck-Institut fur Radioastronomie," Infra. Phys. Technol., vol. 40, no. 3, pp. 191-197, 1999. 
[8] M. R. Swain et al., "Design of the South Pole imaging Fabrey-Perot interferometer (SPIFI)," in Infra. Astron. Instrumentation, Proceedings of Spie, vol. 3354, Kona, Hawaii, March 1998, pp. 480-492.

[9] T. R. Hunter, D. J. Benford, and E. Serabyn, "Optical design of the submillimeter high angular resolution camera (SHARC)," Publ. Astron. Soc. Pacific, vol. 108, no. 729, pp. 1042-1050, 1996

[10] D. J. Benford, E. Serabyn, T. G. Phillips, and S. H. Moseley, "Development of a broadband submillimeter grating spectrometer," in Proc. SPIE Advanced Technology MMW, Radio, and THz Telescopes, vol. 3357, Kona, HI, Mar. 1998, pp. 278-288.

[11] J. J. Bock et al., "Silicon nitride micromesh bolometer arrays for SPIRE," in Proc. SPIE Advanced Technology MMW, Radio, and THz Telescopes, vol. 3357, Kona, HI, Mar. 1998, pp. 297-304.

[12] R. Padman and J. A. Murphy, "Radiation patterns of 'scalar' lightpipes," Infra. Phys., vol. 31, no. 5, pp. 441-446, 1991.

[13] J. A. Murphy, "Radiation patterns of few-moded horns and condensing lightpipes," Infra. Phys., vol. 31, no. 3, pp. 291-299, 1991.

[14] J. Keene, R. H. Hildebrand, S. E. Whitcomb, and R. Winston, "Compact infrared heat trap field optics," Appl. Opt., vol. 17, pp. 1107-1109, 1978.

[15] D. A. Harper, R. H. Hildebrand, R. Stiening, and R. Winston, "Heat trap: An optimized far infrared field optics system," Appl. Opt., vol. 15, pp. $53-59,1976$.

[16] A. Wexler, "Solution of waveguide discontinuities by modal analysis," IEEE Trans. Microwave Theory Tech., vol. MTT-15, Sept. 1967.

[17] A. D. Olver, P. J. B. Clarricoats, A. A. Kishk, and L. Shafai, "Microwave horns and feeds," in Electromagnetic Waves . New York: IEEE Press, 1994, ch. 4.

[18] P. F. Goldsmith, "Quasioptical techniques at millimeter and submillimeter wavelengths," in Infrared and Millimeter Waves, K. J. Button, Ed. New York: Academic, 1982, vol. 6, ch. 5.

[19] S. Withington and G. Yassin, "Modal analysis of partially coherent submillimeter-wave quasioptical systems," IEEE Trans. Antennas Propagat., vol. 46, pp. 1651-1659, Nov. 1998.

[20] M. Born and E. Wolf, Principles of Optics. Oxford, U.K.: Pergamon, 1980.

[21] L. Mandel and E. Wolf, Optical Coherence and Quantum Optics. Cambridge, U.K.: Cambridge Univ. Press, 1995, ch. 6.

[22] C.-T. Tai, "Dyadic Green functions in electromagnetic theory," in Series on Electromagnetic Waves. Piscataway, NJ: IEEE Press, 1994.

[23] G. Toraldo Di Francia, "Degrees of freedom of an image," J. Opt. Soc. Amer., vol. 59, pp. 799-804, 1969.

[24] M. Bedinelli, A. Consortini, L. Ronchi, and B. R. Frieden, "Degrees of freedom, and eigenfunctions for the noisy image," J. Opt. Soc. Amer., vol. 64, pp. 1498-1502, 1974.

[25] L. Mandel and E. Wolf, Optical Coherence and Quantum Optics. Cambridge, U.K.: Cambridge Univ. Press, 1995, pp. 342-349.

[26] E. Wolf, "New theory of partial coherence in the space-frequency domain. Part I: Spectra and cross spectra of steady-state sources," J. Opt. Soc. Amer. A., vol. 72, no. 3, pp. 343-351, 1982.
[27] - "New theory of partial coherence in the space-frequency domain. Part II: Steady-state fields and high-order correlations," J. Opt. Soc. Amer. A., vol. 3, no. 1, pp. 76-85, 1986.

[28] C. O'Sullivan, private communication.

[29] H. Gamo, "Matrix treatment of partial coherence," in Progress in Optics. Amsterdam, the Netherlands: North-Holland, 1964, vol. III, pp. 233-243.

[30] R. H. Clarke and J. Brown, Diffraction Theory and Antennas. Chichester, UK: Ellis Horwood, 1980.

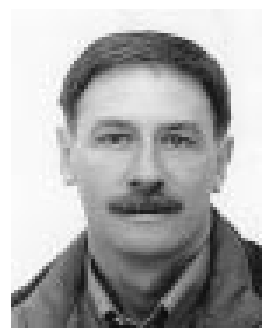

Stafford Withington (M'89) is an Assistant Director of Research in the Astrophysics Group, Cavendish Laboratory, University of Cambridge, U.K. His main area of interest is in the submillimeter-wave band, where he works on optics, detectors, and low-noise instrumentation for astronomy.

Dr. Withington is a Fellow of Downing College, Cambridge.

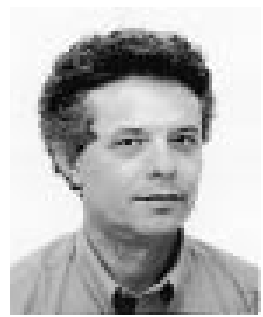

Ghassan Yassin (M'99) received the B.Sc. degree in mathematics and the M.Sc. degree in applied physics from Hebrew University, Jerusalem, Israel, in 1973 and 1977, respectively, and the Ph.D. degree in physics from Keele University, Staffordshire, U.K., in 1981.

He is a Senior Research Associate in the Astrophsyics Group, Cavendish Laboratory, University of Cambridge, Cambridge, U.K. His main research interest is in the areas of superconducting submillimeter-wave detectors and terahertz optics.

J. Anthony Murphy (M'88) received the B.Sc. and M.Sc. degrees in experimental physics from University College Cork, Ireland, in 1977, the M.S. degree in physics from the California Institute of Technology, Pasadena, in 1981, and the Ph.D. degree from Cambridge University, Cambridge, U.K., in 1986.

He is currently a Senior Lecturer and Acting Head of the Experimental Physics Department at the National University of Ireland, Maynooth. His main research interests are in submillimeter-wave optics and receiver development for terrestrial and space telescope systems. 